Korean J. Math. 21 (2013), No. 2, pp. 101-116

http://dx.doi.org/10.11568/kjm.2013.21.2.101

\title{
FUZZY CONNECTIONS AND COMPLETENESS IN COMPLETE RESIDUATED LATTICES
}

\author{
Yong Chan Kim*and Young Sun Kim
}

\begin{abstract}
In this paper, we investigate the properties of fuzzy Galois (dual Galois, residuated, dual residuated) connections in a complete residuated lattice $L$.
\end{abstract}

\section{Introduction}

Galois connection is an important mathematical tool for algebraic structure, data analysis and knowledge processing [1-5,7-11]. Orlowska and Rewitzky [9] investigated the algebraic structures of operators of Galois-style (Galois, dual Galois, residuated, dual residuated) connections on set. Hájek [6] introduced a complete residuated lattice $L$ which is an algebraic structure for many valued logic. Recently, Yao and $\mathrm{Lu}$ [11] introduced Galois connections and fuzzy completeness in a complete residuated lattice $L$. Bělohlávek [1-3] developed the notion of fuzzy contexts using Galois connections with $R \in L^{X \times Y}$ on a complete residuated lattice $L$.

In this paper, we investigate the properties of fuzzy Galois (dual Galois, residuated, dual residuated) connections as an extension of Yao and $\mathrm{Lu}[11]$ in a complete residuated lattice $L$.

Received February 4, 2013. Revised March 29, 2013. Accepted April 10, 2013.

2010 Mathematics Subject Classification: 06A06, 06A15, 06B30, 54F05,68U35.

Key words and phrases: Fuzzy Galois (dual Galois, residuated, dual residuated) connections, Order preserving (reversing) map, Fuzzy complete.

*Corresponding author.

(C) The Kangwon-Kyungki Mathematical Society, 2013.

This is an Open Access article distributed under the terms of the Creative commons Attribution Non-Commercial License (http://creativecommons.org/licenses/by -nc/3.0/) which permits unrestricted non-commercial use, distribution and reproduction in any medium, provided the original work is properly cited. 


\section{Preliminaries}

Definition 2.1. ([6,11]) An algebra $(L, \wedge, \vee, \odot, \rightarrow, 0,1)$ is called a complete residuated lattice if it satisfies the following conditions:

(C1) $L=(L, \leq, \vee, \wedge, 1,0)$ is a complete lattice with the greatest element 1 and the least element 0 ;

(C2) $(L, \odot, 1)$ is a commutative monoid;

(C3) $x \odot y \leq z$ iff $x \leq y \rightarrow z$ for $x, y, z \in L$.

REMARK 2.2. ([6]) (1) A completely distributive lattice $L=(L, \leq$ $, \vee, \wedge=\odot, \rightarrow, 1,0)$ is a complete residuated lattice defined by

$$
x \rightarrow y=\bigvee\{z \mid x \wedge z \leq y\} .
$$

In particular, the unit interval $([0,1], \vee, \wedge=\odot, \rightarrow, 0,1)$ is a complete residuated lattice defined by

$$
x \rightarrow y=\bigvee\{z \mid x \wedge z \leq y\} .
$$

(2) The unit interval $([0,1], \vee, \wedge, \odot, \rightarrow, 0,1)$ with a left-continuous $t$ norm $\odot$ is a complete residuated lattice defined by

$$
x \rightarrow y=\bigvee\{z \mid x \odot z \leq y\} .
$$

In this paper, we assume $(L, \wedge, \vee, \odot, \rightarrow, 0,1)$ is a complete residuated latttice.

Definition 2.3. ([11]) Let $X$ be a set. A function $e_{X}: X \times X \rightarrow L$ is called:

(E1) reflexive if $e_{X}(x, x)=1$ for all $x \in X$,

(E2) transitive if $e_{X}(x, y) \odot e_{X}(y, z) \leq e_{X}(x, z)$, for all $x, y, z \in X$.

(E3) if $e_{X}(x, y)=e_{X}(y, x)=1$, then $x=y$.

If $e_{X}$ satisfies (E1) and (E2), $e_{X}$ is a fuzzy preorder and $\left(X, e_{X}\right)$ is a fuzzy preorder set. If e satisfies (E1), (E2) and (E3), $e_{X}$ is a fuzzy partially order and $\left(X, e_{X}\right)$ is a fuzzy partially order set (simply, fuzzy poset).

EXAMPLE 2.4. (1) We define a function $e_{L}: L \times L \rightarrow L$ as $e_{L}(x, y)=$ $x \rightarrow y$. Then $e_{L}$ is a partial order.

(2) We define a function $e_{L^{X}}: L^{X} \times L^{X} \rightarrow L$ as $e_{L^{X}}(A, B)=$ $\bigwedge_{x \in X}(A(x) \rightarrow B(x))$. Then $\left(L^{X}, e_{L^{X}}\right)$ is a fuzzy poset.

(3) If $\left(X, e_{X}\right)$ is a fuzzy poset and we define a function $e_{X}^{-1}(x, y)=$ $e_{X}(y, x)$, then $\left(X, e_{X}^{-1}\right)$ is a fuzzy poset. 
Definition 2.5. Let $\left(X, e_{X}\right)$ and $\left(Y, e_{Y}\right)$ be a fuzzy poset and $f$ : $X \rightarrow Y$ and $g: Y \rightarrow X$ maps.

(1) $\left(e_{X}, f, g, e_{Y}\right)$ is called a Galois connection if for all $x \in X, y \in Y$,

$$
e_{Y}(y, f(x))=e_{X}(x, g(y)) \text {. }
$$

(2) $\left(e_{X}, f, g, e_{Y}\right)$ is called a dual Galois connection if for all $x \in X, y \in$ $Y$

$$
e_{Y}(f(x), y)=e_{X}(g(y), x) .
$$

(3) $\left(e_{X}, f, g, e_{Y}\right)$ is called a residuated connection if for all $x \in X, y \in$ $Y$

$$
e_{Y}(f(x), y)=e_{X}(x, g(y)) \text {. }
$$

(4) $\left(e_{X}, f, g, e_{Y}\right)$ is called a dual residuated connection if for all $x \in$ $X, y \in Y$,

$$
e_{Y}(y, f(x))=e_{X}(g(y), x) .
$$

(5) $f$ is an order preserving map if $e_{Y}\left(f\left(x_{1}\right), f\left(x_{2}\right)\right) \geq e_{X}\left(x_{1}, x_{2}\right)$ for all $x_{1}, x_{2} \in X$.

(6) $f$ is an order reversing map if $e_{Y}\left(f\left(x_{1}\right), f\left(x_{2}\right)\right) \geq e_{X}\left(x_{2}, x_{1}\right)$ for all $x_{1}, x_{2} \in X$.

\section{Fuzzy connections and completeness in complete residu- ated lattices}

Definition 3.1. ([10]) Let $\left(X, e_{X}\right)$ be a fuzzy poset and $A \in L^{X}$.

(1) A point $x_{0}$ is called a join (or supremum) of $A$, denoted by $x_{0}=\sqcup A$, if it satisfies

(J1) $A(x) \leq e_{X}\left(x, x_{0}\right)$,

$(\mathrm{J} 2) \bigwedge_{x \in X}\left(A(x) \rightarrow e_{X}(x, y)\right) \leq e_{X}\left(x_{0}, y\right)$.

A point $x_{1}$ is called a meet (or infimum) of $A$, denoted by $x_{1}=$ $\sqcap A$, if it satisfies

(M1) $A(x) \leq e_{X}\left(x_{1}, x\right)$,

(M2) $\bigwedge_{x \in X}\left(A(x) \rightarrow e_{X}(y, x)\right) \leq e_{X}\left(y, x_{1}\right)$.

The pair $\left(X, e_{X}\right)$ is called a fuzzy complete lattice if for all $A \in$ $L^{X}, \sqcup A$ and $\sqcap A$ exist.

(2) $x_{0}=\max A$ is called a maximal element if $A\left(x_{0}\right)=1$ and $A(y) \leq$ $e_{X}\left(y, x_{0}\right)$, for all $y \in X$.

(3) $x_{1}=\min A$ is called a minimal element if $A\left(x_{1}\right)=1$ and $A(y) \leq$ $e_{X}\left(x_{1}, y\right)$, for all $y \in X$. 
REMARK 3.2. Let $\left(X, e_{X}\right)$ be a fuzzy poset and $A \in L^{X}$. If $x_{0}$ is a join of $A$, then it is unique because $e_{X}\left(x_{0}, y\right)=e_{X}\left(y_{0}, y\right)$ for all $y \in X$, put $y=x_{0}$ or $y=y_{0}$, then $e_{X}\left(x_{0}, y_{0}\right)=e_{X}\left(y_{0}, x_{0}\right)=1$ implies $x_{0}=y_{0}$. Similarly, if a meet of $A$ exist, then it is unique.

Theorem 3.3. Let $\left(X, e_{X}\right)$ be a fuzzy poset and $A \in L^{X}$.

(1) $x_{0}$ is a join of $A$ iff $\bigwedge_{x \in X}\left(A(x) \rightarrow e_{X}(x, y)\right)=e_{X}\left(x_{0}, y\right)$.

(2) $x_{1}$ is a meet of $A$ iff $\bigwedge_{x \in X}\left(A(x) \rightarrow e_{X}(y, x)\right)=e_{X}\left(y, x_{1}\right)$.

(3) $x_{0}=\max A$ iff $A\left(x_{0}\right)=1$ and $x_{0}=\sqcup A$.

(4) $x_{1}=\min A$ iff $A\left(x_{1}\right)=1$ and $x_{1}=\sqcap A$.

Proof. (1) $(\Rightarrow)$ Let $x_{0}$ be a join of $A$. Then $A(x) \leq e_{X}\left(x, x_{0}\right)$. Thus, $A(x) \odot e_{X}\left(x_{0}, y\right) \leq e_{X}\left(x, x_{0}\right) \odot e_{X}\left(x_{0}, y\right) \leq e_{X}(x, y)$. Hence $e_{X}\left(x_{0}, y\right) \leq$ $\bigwedge_{x \in X}\left(A(x) \rightarrow e_{X}(x, y)\right)$. By (J2), the equality holds.

$(\Leftarrow)$ Since $\bigwedge_{x \in X}\left(A(x) \rightarrow e_{X}\left(x, x_{0}\right)\right)=e_{X}\left(x_{0}, x_{0}\right)=1$, then $A(x) \leq$ $e_{X}\left(x, x_{0}\right)$. Hence the result holds.

(2) $(\Rightarrow)$ Let $x_{1}$ be a meet of $A$. Then $A(x) \leq e_{X}\left(x_{1}, x\right)$. Thus, $e_{X}\left(y, x_{1}\right) \odot A(x) \leq e_{X}\left(y, x_{1}\right) \odot e_{X}\left(x_{1}, x\right) \leq e_{X}(y, x)$. Hence $e_{X}\left(y, x_{1}\right) \leq$ $\bigwedge_{x \in X}\left(A(x) \rightarrow e_{X}(x, y)\right)$. By (M2), the equality holds.

$(\Leftarrow)$ Since $\bigwedge_{x \in X}\left(A(x) \rightarrow e_{X}\left(x_{1}, x\right)\right)=e_{X}\left(x_{1}, x_{1}\right)=1$, then $A(x) \leq$ $e_{X}\left(x_{1}, x\right)$. Hence the result holds.

(3) Let $x_{0}=\max A$. Then

$$
\begin{gathered}
\bigwedge_{z \in X}\left(A(z) \rightarrow e_{X}(z, x)\right) \leq A\left(x_{0}\right) \rightarrow e_{X}\left(x_{0}, x\right)=e_{X}\left(x_{0}, x\right), \\
\bigwedge_{z \in X}\left(A(z) \rightarrow e_{X}(z, x)\right) \geq \bigwedge_{z \in X}\left(e_{X}\left(z, x_{0}\right) \rightarrow e_{X}(z, x)\right)=e_{X}\left(x_{0}, x\right) .
\end{gathered}
$$

Thus $e_{X}\left(x_{0}, x\right)=\bigwedge_{z \in X}\left(A(z) \rightarrow e_{X}(z, x)\right)$. So, $x_{0}=\sqcup A$.

Let $A\left(x_{0}\right)=1$ and $x_{0}=\sqcup A$. Then $e_{X}\left(x_{0}, x_{0}\right)=\bigwedge_{z \in X}(A(z) \rightarrow$ $\left.e_{X}\left(z, x_{0}\right)\right)=1$ implies $A(z) \leq e_{X}\left(z, x_{0}\right)$. Then $x_{0}=\max A$.

(4) It is similarly proved as (3).

Remark 3.4. Let $\left(X, e_{X}\right)$ be a fuzzy poset and $A \in L^{X}$.

(1) Since $\bigwedge_{x \in X}\left(e_{X}(x, y) \rightarrow e_{X}(x, z)\right)=e_{X}(y, z)$, then, by Theorem 3.3 (1), $y=\sqcup\left(e_{X}\right)^{y}$ where $\left(e_{X}\right)^{y}(x)=e_{X}(x, y)$.

(2) Since $\bigwedge_{z \in X}\left(e_{X}(y, z) \rightarrow e_{X}(x, z)\right)=e_{X}(x, y)$, then, by Theorem 3.3 (3), $y=\sqcap\left(e_{X}\right)_{y}$ where $\left(e_{X}\right)_{y}(x)=e_{X}(y, x)$.

Remark 3.5. Let $\left(L, e_{L}\right)$ be a fuzzy poset and $A \in L$. 
(1) If $x_{0}$ is a join of $A$, then $\bigwedge_{x \in L}\left(A(x) \rightarrow e_{L}(x, y)\right)=\bigwedge_{x \in L}(A(x) \rightarrow$ $(x \rightarrow y))=\bigvee_{x \in L}(x \odot A(x)) \rightarrow y=e_{L}\left(x_{0}, y\right)=x_{0} \rightarrow y$. So, $x_{0}=\sqcup A=\bigvee_{x \in L}(x \odot A(x))$.

(2) If $x_{1}$ is a meet of $A$ iff $\bigwedge_{x \in L}\left(A(x) \rightarrow e_{L}(y, x)\right)=\bigwedge_{x \in L}(A(x) \rightarrow$ $(y \rightarrow x))=y \rightarrow \bigwedge_{x \in L}(A(x) \rightarrow x)=e_{X}\left(y, x_{1}\right)=y \rightarrow x_{1}$, then $x_{1}=\sqcap A=\bigwedge_{x \in L}(A(x) \rightarrow x)$.

Example 3.6. Let $X=\{a, b, c\}$ be a set. Define a binary operation $\odot$ (called Eukasiewicz conjection) on $L=[0,1]$ by

$$
x \odot y=\max \{0, x+y-1\}, x \rightarrow y=\min \{1-x+y, 1\} .
$$

Let $\left(X=\{a, b, c\}, e_{X}\right)$ be a fuzzy poset as follows:

$$
\begin{aligned}
& e_{X}(a, a)=1, \quad e_{X}(a, b)=0.6, \quad e_{X}(a, b)=0.5 \\
& e_{X}(b, a)=0.8, \quad e_{X}(b, b)=1, \quad e_{X}(b, c)=0.7 \\
& e_{X}(c, a)=0.9, \quad e_{X}(c, b)=0.6, \quad e_{X}(c, c)=1 .
\end{aligned}
$$

(1) For $A=\left(e_{X}\right)^{a}=(1,0.8,0.9)^{t}$, we have $a=\sqcup A=\max A$ from

$$
\bigwedge\left(A(x) \rightarrow e_{X}(x, z)\right)=e_{X}(a, z) .
$$

(2) For $A=\left(e_{X}\right)^{b}=(0.6,1,0.6)^{t}$, we have $b=\sqcup A=\max A$ from

$$
\bigwedge\left(A(x) \rightarrow e_{X}(x, z)\right)=e_{X}(b, z) .
$$

(3) For $A=\left(e_{X}\right)^{c}=(0.5,0.7,1)^{t}$, we have $c=\sqcup A$ from

$$
\bigwedge_{x \in X}\left(A(x) \rightarrow e_{X}(x, y)\right)=e_{X}(\sqcup A, y)=e_{X}(c, y) .
$$

(4) For $A=\left(e_{X}\right)_{a}=(1,0.6,0.5)^{t}$, we have $a=\sqcap A=\min A$ from

$$
\bigwedge\left(A(x) \rightarrow e_{X}(z, x)\right)=e_{X}(z, a) .
$$

(5) For $A=\left(e_{X}\right)_{b}=(0.8,1,0.7)^{t}$, we have $b=\sqcap A=\min A$ from

$$
\bigwedge\left(A(x) \rightarrow e_{X}(z, x)\right)=e_{X}(z, b) .
$$

(6) For $A=\left(e_{X}\right)_{c}=(0.9,0.6,1)^{t}$, we have $c=\sqcap A=\min A$ from

$$
\bigwedge_{x \in X}\left(A(x) \rightarrow e_{X}(z, x)\right)=e_{X}(z, c) .
$$


(7) For $A=(0.5,0.8,0.6)^{t}, \sqcap A$ and $\sqcup A$ do not exist from:

$$
\begin{aligned}
0.9 & =\bigwedge\left(A(x) \rightarrow e_{X}(x, c)\right) \neq e_{X}(y, c), \forall y \in\{a, b, c\} \\
0.8 & =\bigwedge\left(A(x) \rightarrow e_{X}(a, x)\right) \neq e_{X}(a, y), \forall y \in\{a, b, c\} .
\end{aligned}
$$

Hence $\left(X, e_{X}\right)$ is not fuzzy complete.

Theorem 3.7. Let $\left(X, e_{X}\right)$ and $\left(Y, e_{Y}\right)$ be complete.

(1) $\left(e_{X}, f, g, e_{Y}\right)$ is a Galois connection iff $f$ is an order reversing map and $g(y)=\max f^{\leftarrow}\left(\left(e_{Y}\right)_{y}\right)$ iff $g$ is an order reversing map and $f(x)=\max g^{\leftarrow}\left(\left(e_{X}\right)_{x}\right)$.

(2) $\left(e_{X}, f, g, e_{Y}\right)$ is a dual Galois connection iff $f$ is an order reversing map and $g(y)=\min f^{\leftarrow}\left(\left(e_{Y}\right)^{y}\right)$ iff $g$ is an order reversing map and $f(x)=\min g^{\leftarrow}\left(\left(e_{X}\right)^{x}\right)$.

(3) $\left(e_{X}, f, g, e_{Y}\right)$ is a residuated connection iff $f$ is an order preserving map and $g(y)=\max f^{\leftarrow}\left(\left(e_{Y}\right)^{y}\right)$ iff $g$ is an order preserving map and $f(x)=\min g^{\leftarrow}\left(\left(e_{X}\right)_{x}\right)$.

(4) $\left(e_{X}, f, g, e_{Y}\right)$ is a dual residuated connection iff $f$ is an order preserving map and $g(y)=\min f^{\leftarrow}\left(\left(e_{Y}\right)_{y}\right)$ iff $g$ is an order preserving map and $f(x)=\max g^{\leftarrow}\left(\left(e_{X}\right)^{x}\right)$.

Proof. (1) We only show that $\left(e_{X}, f, g, e_{Y}\right)$ is a Galois connection iff $f$ is an order reversing map and $g(y)=\max f^{\leftarrow}\left(\left(e_{Y}\right)_{y}\right)$ because other case is similarly proved.

$(\Rightarrow)$ Since $e_{X}(x, g(f(x)))=e_{Y}(f(x), f(x))=1$, we have

$$
\begin{aligned}
e_{Y}(f(x), f(y)) & =e_{X}(y, g(f(x))) \\
& \geq e_{X}(y, x) \odot e_{X}(x, g(f(x)))=e_{X}(y, x) .
\end{aligned}
$$

Hence $f$ is an order reversing map. Moreover, $g(y)=\max f^{\leftarrow}\left(\left(e_{Y}\right)_{y}\right)$ because

$$
\begin{aligned}
& f^{\leftarrow}\left(\left(e_{Y}\right)_{y}\right)(g(y))=\left(e_{Y}\right)_{y}(f(g(y)) \\
& =e_{Y}(y, f(g(y))) \\
& =e_{X}(g(y), g(y))=1 \text {, } \\
& f^{\leftarrow}\left(\left(e_{Y}\right)_{y}\right)(x)=\left(e_{Y}\right)_{y}(f(x))=e_{Y}(y, f(x))=e_{X}(x, g(y)) . \\
& (\Leftarrow) \text { Since } g(y)=\max f^{\leftarrow}\left(\left(e_{Y}\right)_{y}\right) \text {, we have } \\
& e_{Y}(y, f(x))=\left(e_{Y}\right)_{y}(f(x))=f^{\leftarrow}\left(\left(e_{Y}\right)_{y}\right)(x) \leq e_{X}(x, g(y)) .
\end{aligned}
$$


Since $g(y)=\max f^{\leftarrow}\left(\left(e_{Y}\right)_{y}\right)$,

$$
\begin{gathered}
f^{\leftarrow}\left(\left(e_{Y}\right)_{y}\right)(g(y))=\left(e_{Y}\right)_{y}\left(f(g(y))=e_{Y}(y, f(g(y)))=1 .\right. \\
e_{X}(x, g(y)) \leq e_{Y}(f(g(y)), f(x)) \odot e_{Y}(y, f(g(y))) \leq e_{Y}(y, f(x)) .
\end{gathered}
$$

Thus $e_{X}(x, g(y))=e_{Y}(y, f(x))$.

(2) We only show that $\left(e_{X}, f, g, e_{Y}\right)$ is a dual Galois connection iff $f$ is an order reversing map and $g(y)=\min f^{\leftarrow}\left(\left(e_{Y}\right)^{y}\right)$ because other case is similarly proved.

$(\Rightarrow)$ Since $e_{X}(g(f(x)), x)=e_{Y}(f(x), f(x))=1$, we have

$$
\begin{aligned}
e_{Y}(f(x), f(y)) & =e_{X}(g(f(y)), x) \\
& \geq e_{X}(g(f(y)), y) \odot e_{X}(y, x)=e_{X}(y, x) .
\end{aligned}
$$

Hence $f$ is an order reversing map. Moreover, $g(y)=\min f^{\leftarrow}\left(\left(e_{Y}\right)^{y}\right)$ because

$$
\begin{aligned}
f^{\leftarrow}\left(\left(e_{Y}\right)^{y}\right)(g(y)) & =\left(e_{Y}\right)^{y}(f(g(y)) \\
& =e_{Y}(f(g(y)), y)=e_{X}(g(y), g(y))=1 \\
f^{\leftarrow}\left(\left(e_{Y}\right)^{y}\right)(x) & =\left(e_{Y}\right)^{y}(f(x))=e_{Y}(f(x), y)=e_{X}(g(y), x) .
\end{aligned}
$$

$(\Leftarrow)$ Since $g(y)=\min f^{\leftarrow}\left(\left(e_{Y}\right)^{y}\right)$, we have

$$
e_{Y}(f(x), y)=\left(e_{Y}\right)^{y}(f(x))=f^{\leftarrow}\left(\left(e_{Y}\right)^{y}\right)(x) \leq e_{X}(g(y), x) .
$$

Since $g(y)=\min f^{\leftarrow}\left(\left(e_{Y}\right)^{y}\right)$,

$$
\begin{gathered}
f^{\leftarrow}\left(\left(e_{Y}\right)^{y}\right)(g(y))=\left(e_{Y}\right)^{y}\left(f(g(y))=e_{Y}(f(g(y)), y)=1 .\right. \\
e_{X}(g(y), x) \leq e_{Y}(f(x), f(g(y))) \odot e_{Y}(f(g(y)), y) \leq e_{Y}(f(x), y) .
\end{gathered}
$$

Thus, $e_{Y}(f(x), y)=e_{X}(g(y), x)$.

(3) It follows from Theorem 3.4 in [11].

(4) First, we show that $\left(e_{X}, f, g, e_{Y}\right)$ is a dual residuated connection iff $f$ is an order preserving map and $g(y)=\min f^{\leftarrow}\left(\left(e_{Y}\right)_{y}\right)$.

$(\Rightarrow)$ Since $e_{X}(g(f(x)), x)=e_{Y}(f(x), f(x))=1$, we have

$$
\begin{aligned}
e_{Y}(f(x), f(y)) & =e_{X}(g(f(x)), y) \\
& \geq e_{X}(x, y) \odot e_{X}(g(f(x)), x)=e_{X}(x, y) .
\end{aligned}
$$

We obtain $g(y)=\min f^{\leftarrow}\left(\left(e_{Y}\right)_{y}\right)$ because

$$
\begin{aligned}
f^{\leftarrow}\left(\left(e_{Y}\right)_{y}\right)(g(y)) & =\left(e_{Y}\right)_{y}(f(g(y)))=e_{Y}(y, f(g(y))) \\
& =e_{X}(g(y), g(y))=1, \\
f^{\leftarrow}\left(\left(e_{Y}\right)_{y}\right)(x) & =\left(e_{Y}\right)_{y}(f(x))=e_{Y}(y, f(x)) \\
& =e_{X}(g(y), x) .
\end{aligned}
$$


$(\Leftarrow)$ Since $g(y)=\min f^{\leftarrow}\left(\left(e_{Y}\right)_{y}\right)$, we have

$$
e_{Y}(y, f(x))=\left(e_{Y}\right)_{y}(f(x))=f^{\leftarrow}\left(\left(e_{Y}\right)_{y}\right)(x) \leq e_{Y}(g(y), x) .
$$

Since $g(y)=\min f^{\leftarrow}\left(\left(e_{Y}\right)_{y}\right)$,

$$
\begin{gathered}
f^{\leftarrow}\left(\left(e_{Y}\right)_{y}\right)(g(y))=\left(e_{Y}\right)_{y}\left(f(g(y))=e_{Y}(y, f(g(y)))=1 .\right. \\
e_{X}(g(y), x) \leq e_{Y}(f(g(y)), f(x)) \odot e_{Y}(y, f(g(y))) \leq e_{Y}(y, f(x)) .
\end{gathered}
$$

Hence $e_{X}(g(y), x)=e_{Y}(y, f(x))$.

Second, we show that $\left(e_{X}, f, g, e_{Y}\right)$ is a dual residuated connection iff $g$ is an order preserving map and $f(x)=\max g^{\leftarrow}\left(\left(e_{X}\right)^{x}\right)$.

$(\Rightarrow)$ Since $e_{Y}(y, f(g(y)))=e_{X}(g(y), g(y))=1$, we have

$$
e_{X}(g(x), g(y))=e_{Y}(x, f(g(y))) \geq e_{Y}(x, y) \odot e_{Y}(y, g(f(y)))=e_{Y}(x, y) .
$$

We obtain $f(x)=\max g^{\leftarrow}\left(\left(e_{X}\right)^{x}\right)$ because

$$
\begin{aligned}
g^{\leftarrow}\left(\left(e_{X}\right)^{x}\right)(f(x)) & =\left(e_{X}\right)^{x}(g(f(x)))=e_{Y}(g(f(x)), x) \\
& =e_{Y}(f(x), f(x))=1, \\
g^{\leftarrow}\left(\left(e_{X}\right)^{x}\right)(y) & =\left(e_{X}\right)^{x}(g(y))=e_{X}(g(y), x)=e_{Y}(y, f(x)) .
\end{aligned}
$$

$(\Leftarrow)$ Since $f(x)=\max g^{\leftarrow}\left(\left(e_{X}\right)^{x}\right)$, we have

$$
e_{X}(g(y), x)=\left(e_{X}\right)^{x}(g(y))=g^{\leftarrow}\left(\left(e_{X}\right)^{x}\right)(y) \leq e_{Y}(y, f(x)) .
$$

Since $f(x)=\max g^{\leftarrow}\left(\left(e_{X}\right)^{x}\right)$,

$$
\begin{gathered}
g^{\leftarrow}\left(\left(e_{X}\right)^{x}\right)(f(x))=\left(e_{X}\right)^{x}\left(g(f(x))=e_{X}(g(f(x)), x)=1 .\right. \\
e_{Y}(y, f(x)) \leq e_{X}(g(y), g(f(x))) \odot e_{X}(g(f(x)), x) \leq e_{X}(g(y), x) .
\end{gathered}
$$

Hence $e_{X}(g(y), x)=e_{Y}(y, f(x))$.

Theorem 3.8. Let $\left(X, e_{X}\right)$ and $\left(Y, e_{Y}\right)$ be complete.

(1) $\left(e_{X}, f, g, e_{Y}\right)$ is a Galois connection iff $f(\sqcup A)=\sqcap f^{\rightarrow}(A)$ for all $A \in L^{X}$ iff $g(\sqcup B)=\sqcap g^{\rightarrow}(B)$ for all $B \in L^{Y}$.

(2) $\left(e_{X}, f, g, e_{Y}\right)$ is a dual Galois connection iff $f(\sqcap A)=\sqcup f \rightarrow(A)$ for all $A \in L^{X}$ iff $g(\sqcap B)=\sqcup g^{\rightarrow}(B)$ for all $B \in L^{Y}$.

(3) $\left(e_{X}, f, g, e_{Y}\right)$ is a residuated connection iff $f(\sqcup A)=\sqcup f^{\rightarrow}(A)$ for all $A \in L^{X}$ iff $g(\sqcap B)=\sqcap g^{\rightarrow}(B)$ for all $B \in L^{Y}$.

(4) $\left(e_{X}, f, g, e_{Y}\right)$ is a dual residuated connection iff, for all $A \in L^{X}$, $f(\sqcap A)=\sqcap f^{\rightarrow}(A)$ iff $g(\sqcup B)=\sqcup g^{\rightarrow}(B)$ for all $B \in L^{Y}$. 
Proof. (1) First, we will show that $\left(e_{X}, f, g, e_{Y}\right)$ is a Galois connection iff $f(\sqcup A)=\sqcap f^{\rightarrow}(A)$ for all $A \in L^{X}$.

$(\Rightarrow)$ Put $y_{0}=\sqcap f^{\rightarrow}(A)$. Then

$$
\begin{aligned}
e_{Y}\left(y, y_{0}\right) & =\bigwedge_{z \in Y}\left(f \rightarrow(A)(z) \rightarrow e_{Y}(y, z)\right) \\
& =\bigwedge_{z \in Y}\left(\left(\bigvee_{f(x)=z} A(x) \rightarrow e_{Y}(y, f(x))\right)\right. \\
& =\bigwedge_{z \in Y} \bigwedge_{f(x)=z}\left(A(x) \rightarrow e_{Y}(y, f(x))\right) \\
& =\bigwedge_{x \in X}\left(A(x) \rightarrow e_{X}(x, g(y))\right) \\
& =e_{X}(\sqcup A, g(y))=e_{Y}(y, f(\sqcup A)) .
\end{aligned}
$$

Hence $y_{0}=f(\sqcup A)=\sqcap f^{\rightarrow}(A)$.

$(\Leftarrow)$ Put $A=\left(e_{X}\right)^{y}$. Since $\sqcup\left(e_{X}\right)^{y}=y$ from Remark 3.4(1), we have $f(y)=f\left(\sqcup\left(e_{X}\right)^{y}\right)=\sqcap f^{\rightarrow}\left(\left(e_{X}\right)^{y}\right)$. By the definition of $\sqcap f^{\rightarrow}\left(\left(e_{X}\right)^{y}\right)$,

$$
e_{Y}(f(y), f(x)) \geq f^{\rightarrow}\left(\left(e_{X}\right)^{y}\right)(f(x))=\bigvee_{f(z)=f(x)}\left(e_{X}\right)^{y}(z) \geq e_{X}(x, y)
$$

Thus, $f$ is order-reversing.

Define $g: Y \rightarrow X$ as $g(y)=\sqcup f^{\leftarrow}\left(\left(e_{Y}\right)_{y}\right)$. By the definition of

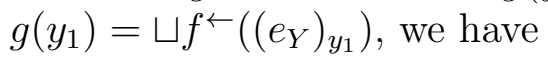

$$
\begin{aligned}
e_{X}\left(g\left(y_{1}\right), g\left(y_{2}\right)\right) & =\bigwedge_{z \in Y}\left(f^{\leftarrow}\left(\left(e_{Y}\right)_{y_{1}}\right)(z) \rightarrow e_{X}\left(z, g\left(y_{2}\right)\right)\right) \\
& \geq \bigwedge_{z \in Y}\left(f^{\leftarrow}\left(\left(e_{Y}\right)_{y_{1}}\right)(z) \rightarrow f^{\leftarrow}\left(\left(e_{Y}\right)_{y_{2}}\right)(z)\right) \\
& =\bigwedge_{z \in Y}\left(e_{Y}\left(y_{1}, f(z)\right) \rightarrow e_{Y}\left(y_{2}, f(z)\right)\right) \\
& \geq e_{Y}\left(y_{2}, y_{1}\right) .
\end{aligned}
$$

Thus, $g$ is order-reversing. Since

$$
f(g(y))=f\left(\sqcup f^{\leftarrow}\left(\left(e_{Y}\right)_{y}\right)\right)=\sqcap f^{\rightarrow}\left(f^{\leftarrow}\left(\left(e_{Y}\right)_{y}\right)\right)
$$




$$
\begin{aligned}
e_{Y}(y, f(g(y))) & =\bigwedge_{z \in Y}\left(f^{\rightarrow}\left(f^{\leftarrow}\left(\left(e_{Y}\right)_{y}\right)\right)(z) \rightarrow e_{Y}(y, z)\right) \\
& =\bigwedge_{z \in Y}\left(\bigvee_{f(x)=z}\left(f^{\leftarrow}\left(\left(e_{Y}\right)_{y}\right)\right)(z) \rightarrow e_{Y}(y, z)\right) \\
& =\bigwedge_{z \in Y}\left(\bigvee_{f(x)=z} e_{Y}(y, f(x)) \rightarrow e_{Y}(y, z)\right) \\
& =\bigwedge_{x \in X}\left(e_{Y}(y, f(x)) \rightarrow e_{Y}(y, f(x))\right)=1 .
\end{aligned}
$$

Since $g(f(x))=\sqcup f^{\leftarrow}\left(\left(e_{Y}\right)_{f(x)}\right)$,

$$
\begin{aligned}
e_{X}(x, g(f(x)) & \geq f^{\leftarrow}\left(\left(e_{Y}\right)_{f(x)}\right)(x)=\left(e_{Y}\right)_{f(x)}(f(x))=1, \\
e_{X}(x, g(y)) & \leq e_{Y}(f(g(y)), f(x))=e_{Y}(f(g(y)), f(x)) \\
& \odot e_{Y}(y, f(g(y))) \leq e_{Y}(y, f(x)), \\
e_{Y}(y, f(x)) & \leq e_{X}(g(f(x)), g(y))=e_{X}(g(f(x)), g(y)) \\
& \odot e_{Y}(x, g(f(x))) \leq e_{X}(x, g(y)) .
\end{aligned}
$$

Thus $e_{X}(x, g(y))=e_{Y}(y, f(x))$.

Second, $\left(e_{X}, f, g, e_{Y}\right)$ is a Galois connection iff $g(\sqcup B)=\sqcap g \rightarrow(B)$ for all $B \in L^{Y}$.

$(\Rightarrow)$ Put $x_{0}=\sqcap g \rightarrow(B)$. Then

$$
\begin{aligned}
e_{X}\left(x, x_{0}\right) & =\bigwedge_{z \in X}\left(g \rightarrow(B)(z) \rightarrow e_{X}(x, z)\right) \\
& =\bigwedge_{z \in X}\left(\bigvee_{g(y)=z} B(y) \rightarrow e_{X}(x, g(y))\right) \\
& =\bigwedge_{z \in X} \bigwedge_{g(y)=z}\left(B(y) \rightarrow e_{Y}(y, f(x))\right) \\
& =\bigwedge_{y \in Y}\left(B(y) \rightarrow e_{Y}(y, f(x))\right) \\
& =e_{Y}\left(\sqcup_{l} B, f(x)\right)=e_{X}\left(x, g\left(\sqcup_{l} B\right)\right) .
\end{aligned}
$$

$(\Leftarrow)$ Put $B=\left(e_{Y}\right)^{y}$. Then $g(y)=g\left(\sqcup\left(e_{Y}\right)^{y}\right)=\sqcap g \rightarrow\left(\left(e_{Y}\right)^{y}\right)$. By the definition of $\sqcap g \rightarrow\left(\left(e_{Y}\right)^{y}\right)$,

$$
e_{X}(g(y), g(w)) \geq g^{\rightarrow}\left(\left(e_{Y}\right)^{y}\right)(g(w))=\bigvee_{g(z)=g(w)}\left(e_{Y}\right)^{y}(z) \geq e_{Y}(w, y) .
$$

Thus, $g$ is order-reversing. 
Define $f: X \rightarrow Y$ as $f(x)=\sqcup g^{\leftarrow}\left(\left(e_{X}\right)_{x}\right)$. By the definition of $f\left(x_{1}\right)=\sqcup g^{\leftarrow}\left(\left(e_{X}\right)_{x_{1}}\right)$, we have

$$
\begin{aligned}
e_{Y}\left(f\left(x_{1}\right), f\left(x_{2}\right)\right) & =\bigwedge_{z \in Y}\left(g^{\leftarrow}\left(\left(e_{X}\right)_{x_{1}}\right)(z) \rightarrow e_{Y}\left(z, f\left(x_{2}\right)\right)\right) \\
& \geq \bigwedge_{z \in Y}\left(g^{\leftarrow}\left(\left(e_{X}\right)_{x_{1}}\right)(z) \rightarrow g^{\leftarrow}\left(\left(e_{X}\right)_{x_{2}}\right)(z)\right) \\
& =\bigwedge_{z \in Y}\left(e_{X}\left(x_{1}, g(z)\right) \rightarrow e_{X}\left(x_{2}, g(z)\right)\right) \\
& \geq e_{X}\left(x_{2}, x_{1}\right) .
\end{aligned}
$$

Thus, $f$ is order-reversing. Since

$$
\begin{aligned}
g(f(x))= & g\left(\sqcup g^{\leftarrow}\left(\left(e_{X}\right)_{x}\right)\right)=\sqcap g^{\rightarrow}\left(g^{\leftarrow}\left(\left(e_{X}\right)_{x}\right)\right) \\
e_{X}(x, g(f(x))) & =\bigwedge_{z \in X}\left(g^{\rightarrow}\left(g^{\leftarrow}\left(\left(e_{X}\right)_{x}\right)\right)(z) \rightarrow e_{X}(x, z)\right) \\
& =\bigwedge_{z \in X}\left(\bigvee_{g(w)=z}\left(g^{\leftarrow}\left(\left(e_{X}\right)_{x}\right)(w) \rightarrow e_{X}(x, z)\right)\right. \\
& =\bigwedge_{z \in X}\left(\bigvee_{g(w)=z} e_{X}(x, g(w)) \rightarrow e_{X}(x, z)\right) \\
& \left.=\bigwedge_{w \in Y}\left(e_{X}(x, g(w))\right) \rightarrow e_{X}(x, g(w))\right)=1
\end{aligned}
$$

Since $f(g(y))=\sqcup g^{\leftarrow}\left(\left(e_{X}\right)_{g(y)}\right)$,

$$
\begin{aligned}
e_{Y}(y, f(g(y)) & \geq g^{\leftarrow}\left(\left(e_{X}\right)_{g(y)}\right)(y)=\left(e_{X}\right)_{g(y)}(g(y))=1, \\
e_{X}(x, g(y)) & \leq e_{Y}(f(g(y)), f(x))=e_{Y}(f(g(y)), f(x)) \\
& \odot e_{Y}(y, f(g(y))) \leq e_{Y}(y, f(x)) \\
e_{Y}(y, f(x)) & \leq e_{X}(g(f(x)), g(y))=e_{X}(g(f(x)), g(y)) \\
& \odot e_{Y}(x, g(f(x))) \leq e_{X}(x, g(y)) .
\end{aligned}
$$

Thus $e_{X}(x, g(y))=e_{Y}(y, f(x))$.

(2) and (3) are similarly proved in (1) and Theorem 3.5 in [11], respectively.

(4) First, $\left(e_{X}, f, g, e_{Y}\right)$ is a dual residuated connection iff $f(\sqcap A)=$ $\sqcap f^{\rightarrow}(A)$ for all $A \in L^{X}$. 
$(\Rightarrow)$ Put $y_{1}=\sqcap f \rightarrow(A)$. Then

$$
\begin{aligned}
e_{Y}\left(y, y_{1}\right) & =\bigwedge_{z \in Y}\left(f \rightarrow(A)(z) \rightarrow e_{Y}(y, z)\right) \\
& =\bigwedge_{x \in X}\left(\bigvee_{f(x)=z} A(x) \rightarrow e_{Y}(y, f(x))\right) \\
& =\bigwedge_{x \in X} \bigwedge_{f(x)=z}\left(A(x) \rightarrow e_{X}(g(y), x)\right) \\
& =\bigwedge_{x \in X}\left(A(x) \rightarrow e_{X}(g(y), x)\right) \\
& =e_{X}(g(y), \sqcap A)=e_{Y}(y, f(\sqcap A)) .
\end{aligned}
$$

Hence $y_{1}=f(\sqcap A)=\sqcap f \rightarrow(A)$.

$(\Leftarrow)$ Put $A=\left(e_{X}\right)_{x}$. Since $\sqcap\left(e_{X}\right)_{x}=x$, then $f(x)=f\left(\sqcap\left(e_{X}\right)_{x}\right)=$ $\sqcap f^{\rightarrow}\left(\left(e_{X}\right)_{x}\right)$. By the definition of $\sqcap f^{\rightarrow}\left(\left(e_{X}\right)_{x}\right)$,

$$
e_{Y}(f(x), f(z)) \geq f^{\rightarrow}\left(\left(e_{X}\right)_{x}\right)(f(z))=\bigvee_{f(d)=f(z)}\left(e_{X}\right)_{x}(d) \geq e_{X}(x, z)
$$

Thus, $f$ is an order preserving map.

Define $g: Y \rightarrow X$ as $g(y)=\sqcap f^{\leftarrow}\left(\left(e_{Y}\right)_{y}\right)$. By the definition of $g\left(y_{2}\right)=\sqcap f^{\leftarrow}\left(\left(e_{Y}\right)_{y_{2}}\right)$, we have

$$
\begin{aligned}
e_{X}\left(g\left(y_{1}\right), g\left(y_{2}\right)\right) & =\bigwedge_{z \in X}\left(f^{\leftarrow}\left(\left(e_{Y}\right)_{y_{2}}\right)(z) \rightarrow e_{X}\left(g\left(y_{1}\right), z\right)\right) \\
& \geq \bigwedge_{z \in X}\left(f^{\leftarrow}\left(\left(e_{Y}\right)_{y_{2}}\right)(z) \rightarrow f^{\leftarrow}\left(\left(e_{Y}\right)_{y_{1}}\right)(z)\right) \\
& =\bigwedge_{z \in X}\left(e_{Y}\left(y_{2}, f(z)\right) \rightarrow e_{Y}\left(y_{1}, f(z)\right)\right) \\
& \geq e_{Y}\left(y_{1}, y_{2}\right) .
\end{aligned}
$$

Thus, $g$ is an order preserving map. Since

$$
f(g(y))=f\left(\sqcap f^{\leftarrow}\left(\left(e_{Y}\right)_{y}\right)\right)=\sqcap f^{\rightarrow}\left(f^{\leftarrow}\left(\left(e_{Y}\right)_{y}\right)\right)
$$




$$
\begin{aligned}
e_{Y}(y, f(g(y))) & =\bigwedge_{z \in X}\left(f^{\rightarrow}\left(f^{\leftarrow}\left(\left(e_{Y}\right)_{y}\right)\right)(z) \rightarrow e_{Y}(y, z)\right) \\
& =\bigwedge_{z \in X}\left(\bigvee_{f(x)=z}\left(f^{\leftarrow}\left(\left(e_{Y}\right)_{y}\right)(x) \rightarrow e_{Y}(y, z)\right)\right. \\
& =\bigwedge_{z \in X}\left(\bigvee_{f(x)=z} e_{Y}(y, f(x)) \rightarrow e_{Y}(y, f(x))\right)=1
\end{aligned}
$$

Since $g(f(x))=\sqcap f^{\leftarrow}\left(\left(e_{Y}\right)_{f(x)}\right)$,

$$
\begin{aligned}
e_{X}(g(f(x)), x) \geq f^{\leftarrow}\left(\left(e_{Y}\right)_{f(x)}\right)(x)=\left(e_{X}\right)_{f(x)}(f(x))=1 . \\
e_{X}(g(y), x) \leq e_{Y}(f(g(y)), f(x))=e_{Y}(f(g(y)), f(x)) \\
\odot e_{Y}(y, f(g(y))) \leq e_{Y}(y, f(x)) \\
e_{Y}(y, f(x)) \leq e_{X}(g(y), g(f(x)))=e_{X}(g(y), g(f(x))) \\
\odot e_{Y}(g(f(x)), x) \leq e_{X}(g(y), x) .
\end{aligned}
$$

Thus $e_{X}(g(y), x)=e_{Y}(y, f(x))$.

Second, $\left(e_{X}, f, g, e_{Y}\right)$ is a dual residuated connection iff $g(\sqcup B)=$ $\sqcup g^{\rightarrow}(B)$ for all $B \in L^{Y}$.

$(\Rightarrow)$ Put $x_{0}=\sqcup g \rightarrow(B)$. Then $g(\sqcup B)=\sqcup g \rightarrow(B)$ from:

$$
\begin{aligned}
e_{X}\left(x_{0}, x\right) & =\bigwedge_{z \in X}\left(g \rightarrow(B)(z) \rightarrow e_{X}(z, x)\right) \\
& =\bigwedge_{z \in X}\left(\left(\bigvee_{g(y)=z} B(y) \rightarrow e_{X}(g(y), x)\right)\right. \\
& =\bigwedge_{z \in X} \bigwedge_{g(y)=z}\left(B(y) \rightarrow e_{Y}(y, f(x))\right) \\
& =\bigwedge_{y \in Y}\left(B(y) \rightarrow e_{Y}(y, f(x))\right) \\
& =e_{Y}(\sqcup B, f(x))=e_{X}(g(\sqcup B), x) .
\end{aligned}
$$

Thus, $x_{0}=g(\sqcup B)=\sqcup g \rightarrow(B)$. 
$(\Leftarrow)$ Put $B=\left(e_{Y}\right)^{y}$. Since $\sqcup\left(e_{Y}\right)^{y}=y$, we have $g(y)=g\left(\sqcup\left(e_{Y}\right)^{y}\right)=$ $\sqcup g \rightarrow\left(\left(e_{Y}\right)^{y}\right)$. By the definition of $\sqcup g^{\rightarrow}\left(\left(e_{Y}\right)^{y}\right)$,

$$
\begin{aligned}
e_{X}(g(y), g(z)) & =\bigwedge_{p \in X}\left(g^{\rightarrow}\left(\left(e_{Y}\right)^{y}\right)(p) \rightarrow e_{X}(p, g(z))\right) \\
& =\bigwedge_{p \in X}\left(\bigvee_{g(w)=p}\left(e_{Y}\right)^{y}(w) \rightarrow e_{X}(g(w), g(z))\right) \\
& =\bigwedge_{p \in X} \bigwedge_{g(w)=p}\left(e_{Y}(w, y) \rightarrow e_{X}(g(w), g(z))\right) \\
& =\bigwedge_{w \in Y}\left(e_{Y}(w, y) \rightarrow e_{X}(g(w), g(z))\right) \\
e_{Y}(w, y) & \leq \bigwedge_{z \in Y}\left(e_{X}(g(y), g(z)) \rightarrow e_{X}(g(w), g(z))\right) \\
& =e_{X}(g(w), g(y)) .
\end{aligned}
$$

Thus, $g$ is order-preserving.

Define $f: X \rightarrow Y$ as $f(x)=\sqcup g^{\leftarrow}\left(\left(e_{X}\right)^{x}\right)$. Since $e_{Y}(f(x), w) \leq$ $g^{\leftarrow}\left(\left(e_{X}\right)^{x}\right)(z) \rightarrow e_{Y}(z, w)$,

$$
g^{\leftarrow}\left(\left(e_{X}\right)^{x}\right)(z) \leq \bigwedge_{w \in Y}\left(e_{X}(g(w), x) \rightarrow e_{Y}(z, w)\right)=e_{Y}(z, g(y)) .
$$

Thus, $g$ is order-preserving, by the definition of $f\left(x_{1}\right)=\sqcup g^{\leftarrow}\left(\left(e_{X}\right)^{x_{1}}\right)$,

$$
\begin{aligned}
e_{Y}\left(f\left(x_{1}\right), f\left(x_{2}\right)\right) & =\bigwedge_{z \in X}\left(g^{\leftarrow}\left(\left(e_{X}\right)^{x_{1}}\right)(z) \rightarrow e_{Y}\left(z, f\left(x_{2}\right)\right)\right) \\
& \geq \bigwedge_{z \in X}\left(g^{\leftarrow}\left(\left(e_{X}\right)^{x_{1}}\right)(z) \rightarrow g^{\leftarrow}\left(\left(e_{X}\right)^{x_{2}}\right)(z)\right) \\
& =\bigwedge_{z \in X}\left(e_{X}\left(g(z), x_{1}\right) \rightarrow e_{X}\left(g(z), x_{2}\right)\right) \\
& \geq e_{X}\left(x_{1}, x_{2}\right) .
\end{aligned}
$$


Since $g(f(x))=g\left(\sqcup g^{\leftarrow}\left(\left(e_{X}\right)^{x}\right)\right)=\sqcup g^{\rightarrow}\left(g^{\leftarrow}\left(\left(e_{X}\right)^{x}\right)\right)$, we have

$$
\begin{aligned}
e_{X}(g(f(x)), x) & =\bigwedge_{z \in X}\left(g^{\rightarrow}\left(g^{\leftarrow}\left(\left(e_{X}\right)^{x}\right)\right)(z) \rightarrow e_{X}(z, x)\right) \\
& =\bigwedge_{z \in X}\left(\bigvee_{g(y)=z}\left(g^{\leftarrow}\left(\left(e_{X}\right)^{x}\right)\right)(z) \rightarrow e_{X}(z, x)\right) \\
& =\bigwedge_{z \in X}\left(\bigvee_{g(y)=z} e_{X}(g(y), x) \rightarrow e_{Y}(g(y), x)\right)=1
\end{aligned}
$$

Since $f(g(y))=\sqcup g^{\leftarrow}\left(\left(e_{X}\right)^{g(y)}\right)$,

$$
\begin{aligned}
e_{Y}(y, f(g(y)) & \geq g^{\leftarrow}\left(\left(e_{X}\right)^{g(y)}\right)(y)=\left(e_{X}\right)^{g(y)}(g(y))=1, \\
e_{X}(g(y), x) & \leq e_{Y}(f(g(y)), f(x))=e_{Y}(f(g(y)), f(x)) \\
& \odot e_{Y}(y, f(g(y))) \leq e_{Y}(y, f(x)) \\
e_{Y}(y, f(x)) & \leq e_{X}(g(y), g(f(x)))=e_{X}(g(y), g(f(x))) \\
& \odot e_{Y}(g(f(x)), x) \leq e_{X}(g(y), x) .
\end{aligned}
$$

Thus $e_{X}(g(y), x)=e_{Y}(y, f(x))$.

\section{References}

[1] R. Bělohlávek, Fuzzy Galois connections, Math. Log. Q. 45 (1999), 497-504.

[2] R. Bělohlávek, Lattices of fixed points of Galois connections, Math. Log. Q. 47 (2001), 111-116.

[3] R. Bělohlávek, Concept lattices and order in fuzzy logic, Ann. Pure Appl. Logic 128 (2004), 277-298.

[4] J.G. Garcia, I.M. Perez, M.A. Vicente, D. Zhang, Fuzzy Galois connections categorically, Math. Log. Q. 56 (2) (2010), 131-147.

[5] G. Georgescu, A. Popescue, Non-dual fuzzy connections, Arch. Math. Logic 43 (2004), 1009-1039.

[6] P. Hájek, Metamathematices of Fuzzy Logic, Kluwer Academic Publishers Dordrecht (1998).

[7] J. Järvinen, M. Kondo, J. Kortelainen, Logics from Galois connections, Internat. J. Approx. Reason. 49 (2008), 595-606.

[8] A. Melton, D.A. Schmidt, G.E. Strecker, Galois connections and compter sciences, Leture Notes in Computer Science, Springer-Verlag 240 (1986), 299-312.

[9] Ewa. Orlowska, I. Rewitzky, Algebras for Galois-style connections and their discrete duality, Fuzzy Sets and Systems 161 (2010), 1325-1342.

[10] R. Wille, Restructuring lattice theory; an approach based on hierarchies of concept, in: 1. Rival(Ed.), Ordered Sets, Reidel, Dordrecht, Boston, 1982. 
[11] W. Yao, L.X. Lu, Fuzzy Galois connections on fuzzy posets, Math. Log. Q. 55 (1) (2009), 105-112.

Department of Mathematics

Natural Science

Gangneung-Wonju National University

Gangneung, Gangwondo, 210-702, Korea

E-mail: yck@gwnu.ac.kr

Department of Applied Mathematics

Pai Chai University

Dae Jeon, 302-735, Korea

E-mail: yskim@pcu.ac.kr 